\title{
Comparison of Animal Models with Soft Tissue Infection by Different Bacilli
}

\author{
Wan-Yu LIN ${ }^{1)}$, Shih-Chuan TSAI ${ }^{2)}$, Guang-Uei HUNG ${ }^{3)}$, Po-Cheung KWAN ${ }^{4}$, Chen-Fu LIN ${ }^{5}$, Chia-Suey YUAN ${ }^{5}$ and \\ Yung-Chang $\operatorname{LIN}^{6,7) *}$ \\ ${ }^{1)}$ Departments of Nuclear Medicine, ${ }^{4)}$ Pathology and ${ }^{5)}$ Medicine Laboratory, Taichung Veterans General Hospital, Taichung, \\ ${ }^{2)}$ Department of Nuclear Medicine, Show Chwan Memorial Hospital, Changhua, $\left.{ }^{3}\right)$ Department of Nuclear Medicine, Changhua Christian \\ Hospital, Changhua and ${ }^{6)}$ Veterinary Medical Teaching Hospital and ${ }^{7}$ Department of Veterinary Medicine, College of Veterinary \\ Medicine, National Chung-Hsing University, Taichung, Taiwan
}

(Received 26 March 2004/Accepted 9 September 2004)

\begin{abstract}
In clinical medicine improved diagnostic methods for the detection of infection are needed. A good infectious animal model is very important for the development of a new diagnostic method or drug. The purpose of this study was to establish a good animal model with soft tissue infection. Twenty-four SD rats were divided into four groups (6 in each group). Various bacilli including Staphylococcus aureus (S. aureus), Streptococcus pneumoniae (S. pneumoniae), and Escherichia coli (E. coli) were injected intramuscularly into the left caudal thighs of three groups of rats to create soft tissue infection. In addition, normal saline was injected into the left caudal thighs of ten rats which were used as controls. Before and $48 \mathrm{hr}$ after inoculation of the bacilli, a blood sample $(0.5 \mathrm{~m} l)$ was taken from each rat and analyzed to determine the white blood cell count and differentiated cell count. In addition, $48 \mathrm{hr}$ after the inoculation, 0.2 $\mathrm{mCi}$ of gallium-67 was injected via the tail vein. Gallium scan was performed at $24 \mathrm{hr}$ and $48 \mathrm{hr}$ after administration of the radiotracer. The dorsal view of both hind legs was imaged and analyzed by computers to calculate the lesion-to-normal (L/N) ratio. After imaging, all rats were sacrificed and specimens from portions of the infected thigh muscle were sent for histopathologic investigation to confirm the infection. The increase in both the WBC counts and the segmented polymorphonuclear leukocytes (PMNs) were most significant in the $S$. aureus group, followed by the $S$. pneumoniae group, E. coli group and normal control groups. The rats with $S$. aureus infection had significant gallium uptake at the site of infection and the highest $\mathrm{L} / \mathrm{N}$ ratio of 2.14 on the 24 -hr image and 2.0 on the 48 -hr image. The rats with $S$. pneumoniae had the second highest $\mathrm{L} / \mathrm{N}$ ratio $(1.41 \mathrm{at} 24 \mathrm{hr}$, and 1.48 at $48 \mathrm{hr})$. The $\mathrm{L} / \mathrm{N}$ ratio for the $E$. coli group was 1.27 at $24 \mathrm{hr}$ and 1.35 at $48 \mathrm{hr}$. No obviously abnormal gallium uptake was demonstrated in the normal controls. We conclude that all three bacilli induced a soft tissue infection in SD rats. S. aureus resulted in the most significant infectious signs. KEY WORDS: bacilli, gallium-67 scan, infectious animal model, SD rats.
\end{abstract}

Since a major consideration in the treatment of patients with focal infection is drainage, prompt identification of the site of involvement is extremely important. Early in the evolution of an inflammatory lesion, before formation of an abscess, radiological methods such as plain x-ray, computed tomography and ultrasound cannot readily localize the area of involvement. In addition, in a case with unknown fever, a whole body survey is always needed to locate the infectious source. Radionuclide techniques allow for early diagnosis of infection as well as whole body scanning, and are widely used in the detection of infection in patients with unknown fever. Gallium-67 citrate (gallium) scan and leukocyte scan are the two most common techniques used in nuclear medicine departments but at least $48 \mathrm{hr}$ is required to obtain the final results of the gallium scan, which is a major drawback in its use for the detection of infection. In addition, significant physiological bowel activity in the gallium image becomes an obstacle when applied to patients with suspected intra-abdominal infection $[3,5,10,13,14$, 23]. Labeled leukocytes provide more accurate and quicker diagnosis than gallium imaging but In-111 is not available in many countries, Tc-99m HMPAO labeling of leukocytes

\footnotetext{
* Correspondence to: Lin, Y.-C., Department of Veterinary Medicine, National Chung-Hsing University, 250, Kuo-Kuang Road, Taichung 40227, Taiwan.
}

is expensive and time consuming, and Tc-99m HMPAO scan may also show intense physiologic bowel activity. Again, this limits its application in patients with suspected intra-abdominal infection $[3,14,16,20]$. In addition, Tc$99 \mathrm{~m}$ labeled human IgG have also been used in the detection of infection [7, 21]. Therefore, there is an urgent need in nuclear medicine to develop a new drug or new technique to detect infectious foci $[22,24]$.

In order to develop such a drug or technique it is important to establish a good and reliable animal infectious model. In the literature, different kinds of micro-organisms, including Staphylococcus aureus ( $S$. aureus), Escherichia coli (E. coli), Streptococcus pneumoniae ( $S$. pneumoniae) have been used to produce infectious foci in different animals such as mice, rats and rabbits $[2,4,6,8]$. There are no comparative data on the abilities of these micro-organisms to induce infectious foci. In this study, we evaluated the ability of different kinds of micro-organisms including $S$. aureus, E. coli, and $S$. pneumoniae to induce infectious foci in rats and established a good and reliable infectious animal model.

\section{MATERIALS AND METHODS}

Animals: Twenty-four male Sprague-Dawley (SD) rats (Charles River Breeding Laboratories, Burlington, MD, U.S.A.) weighing $150 \mathrm{~g}$ were used in this experiment, and 
were divided into four groups (six in each group). Single clinical isolates of $S$. aureus, E. coli, and $S$. pneumoniae, were used to induce infectious foci. The appropriate microbial strain was incubated overnight on trypticase soy agar plates at $37^{\circ} \mathrm{C}$. Individual colonies were diluted with sterile normal saline to produce a turbid suspension containing approximately $5 \times 10^{9}$ organisms $/ \mathrm{ml}$. The rats were anesthetized with ketamine, and $0.2 \mathrm{~m} l$ of a suspension containing $1 \times 10^{9}$ organisms was injected into the left caudal thigh of the rats. In addition, the six rats in the control group were injected with $0.2 \mathrm{~m} l$ of normal saline.

\section{Evaluation of infection}

Blood Cell Counts: Before and $48 \mathrm{hr}$ after inoculation with the bacilli, a blood sample $(0.5 \mathrm{ml})$ was taken from each rat and analyzed to determine the white blood cell count (WBC) and segmented polymorphonuclear leukocytes (PMNs).

Gallium-67 scintigraphy: All rats were injected with 0.2 $\mathrm{mCi}$ gallium-67 citrate via the tail vein $24 \mathrm{hr}$ after the injection of bacilli or normal saline into the left caudal thigh. The rats were anaesthetized and tied to a board before imaging, then they were placed in the prone position under the camera with the legs stretched out and fixed. A planar dorsal view of the hind legs with a total count of $300 \mathrm{~K}$ was obtained at 24 and $48 \mathrm{hr}$ after administration of the radioactive agents with a large field-of-view gamma-camera (Elscint APEX $415 \mathrm{~W}$ ) with an APC-3 (low energy, medium resolution, medium sensitivity) collimator. The images were obtained in a $256 \times 256$ matrix.

Calculation of uptake ratio: Regions of interest (ROIs) were drawn over the infected and non-infected thighs to determine radioactivity at the site of infection (Fig. 1). The edge of the ROI over the infected thigh was defined manually when a threshold of $30 \%$ of maximum counts in the lesion was reached. The threshold was easy to define in a color image shown on the computer. The ROI over the noninfected thigh was definted empirically as of a similar size to that of the right thigh. It was not necessary for both ROIs to be exactly the same size since mean counts per pixel were used for calculation. The formula used to calculate the lesion-to-normal ratio (L/ $\mathrm{N}$ ratio) for the ROIs of each rabbit is shown below:

$$
\mathrm{L} / \mathrm{N} \text { ratio }=\frac{\text { Mean counts per pixel of ROI1 }}{\text { Mean counts per pixel of ROI2. }}
$$

Where

ROI1 $=$ ROI over infected caudal thigh

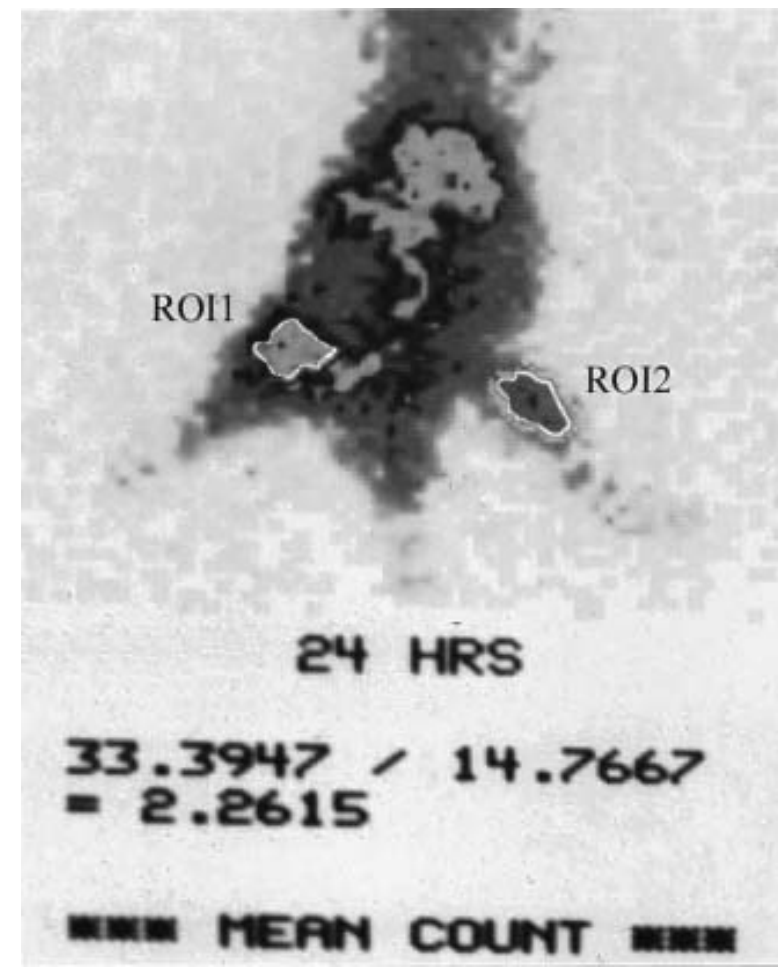

Fig. 1. Dorsal view of a rat injected S. aureus. Regions of interest (ROIs) were drawn over the infected left caudal thigh (ROI1) and non-infected right caudal thigh (ROI2) to generate the mean counts per pixel. The $\mathrm{L} / \mathrm{N}$ ratio was calculated with the following equation: $\mathrm{L} / \mathrm{N}$ ratio $=$ mean counts per pixel of ROI1/ mean counts per pixel of ROI2.

ROI2=ROI over non-infected caudal thigh

The $\mathrm{L} / \mathrm{N}$ ratio was taken as an index of the ability of the radiopharmaceutical to localize the infection.

Pathologic examination: After finishing the gallium imaging, the infected animals were sacrificed and specimens from portions of the infected caudal thigh muscle were sent for histopathologic investigation to confirm the infection.

Statistical analysis: Mann Whitney U test were used for testing differences. The test results were considered statistically significant when $p$ value was $<0.05$. Data were analyzed using the Statistica for Windows Release 4.5 package (StatSoft, Inc., OK, US).

Table 1. Hematologic data after intramuscular injection of various organisms into the left caudal thigh of rats

\begin{tabular}{|c|c|c|c|c|c|c|}
\hline & \multicolumn{3}{|c|}{$\mathrm{WBC}($ count $/ \mathrm{m} l)$} & \multicolumn{3}{|c|}{ PMNs (\%) } \\
\hline & Baseline & $48 \mathrm{hr}$ & $\%$ Increase & Baseline & $48 \mathrm{hr}$ & \% Increase \\
\hline Control & $8124 \pm 903$ & $9024 \pm 1933$ & 11.07 & $18.8 \pm 4.15$ & $17.6 \pm 13.3$ & -1.2 \\
\hline S. aureus & $7638 \pm 1722$ & $12682 \pm 796$ & 66.03 & $10.6 \pm 1.9$ & $39.7 \pm 14.5$ & 29.1 \\
\hline E. coli & $8838 \pm 1656$ & $10816 \pm 1127$ & 22.38 & $15.2 \pm 5.2$ & $14.8 \pm 6.1$ & -0.4 \\
\hline S. pneunoniae & $7024 \pm 1532$ & $10476 \pm 1762$ & 49.14 & $11.2 \pm 3.2$ & $27.2 \pm 9.5$ & 16.0 \\
\hline
\end{tabular}

PMNs: segmented polymorphonuclear leukocytes. 


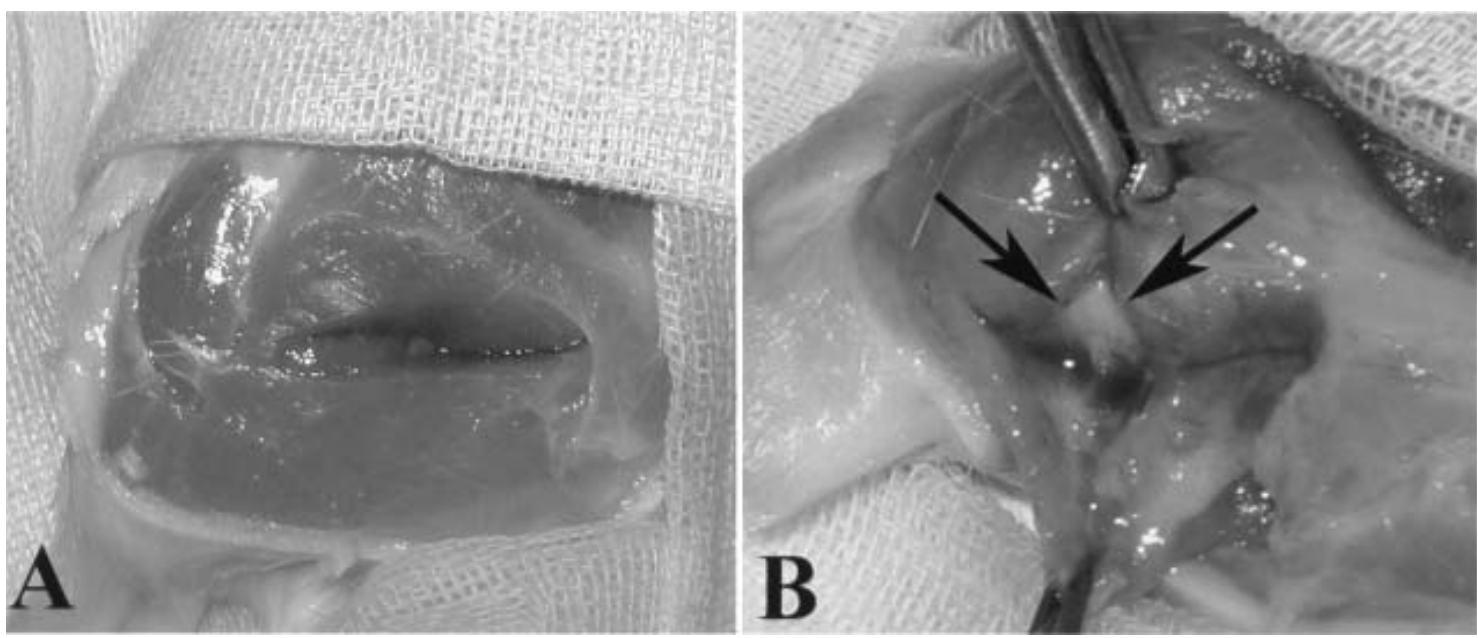

Fig. 2. Macroscopic examination of the injected thigh of the rats. (A) Normal control: No redness, swelling or abscess formation was observed. (B) The rat with S. aureus injection: Abscess formation was observed (arrows).
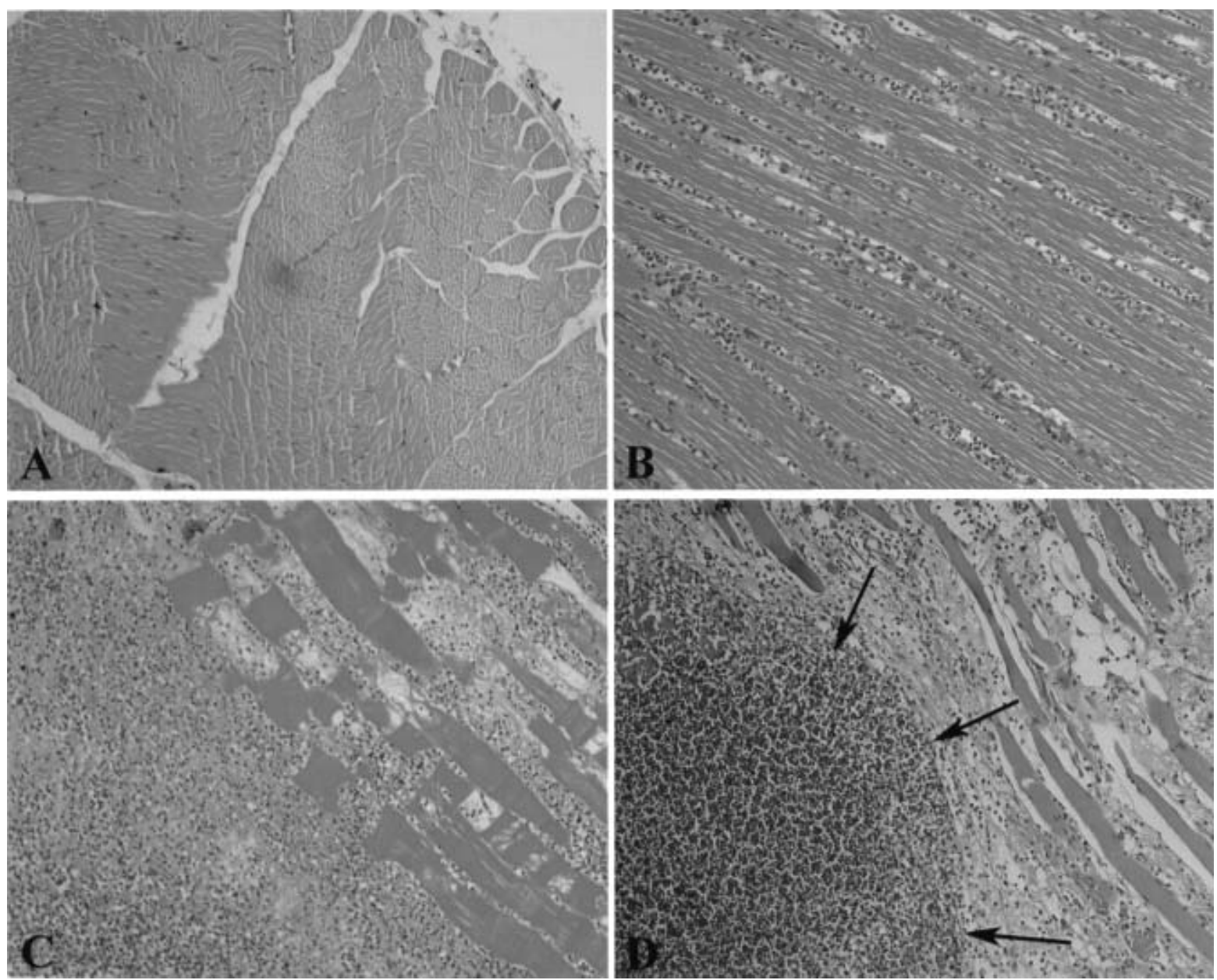

Fig. 3. Microscopic pathologic examination. (HE stain, $100 \times$ )(A) Normal control. Intact muscular structure with no significant inflammatory cells infiltration. (B) E. coli group. Mild inflammatory cells infiltration between the muscle fibers. (C) S. pneumoniae group. Obvious infiltration by inflammatory cells in the muscle with destruction of the muscular structure. (D) S. Aureus group. Significant infiltration of the inflammatory cells with abscess formation (arrows). Destruction of the muscular structure was also significant. 

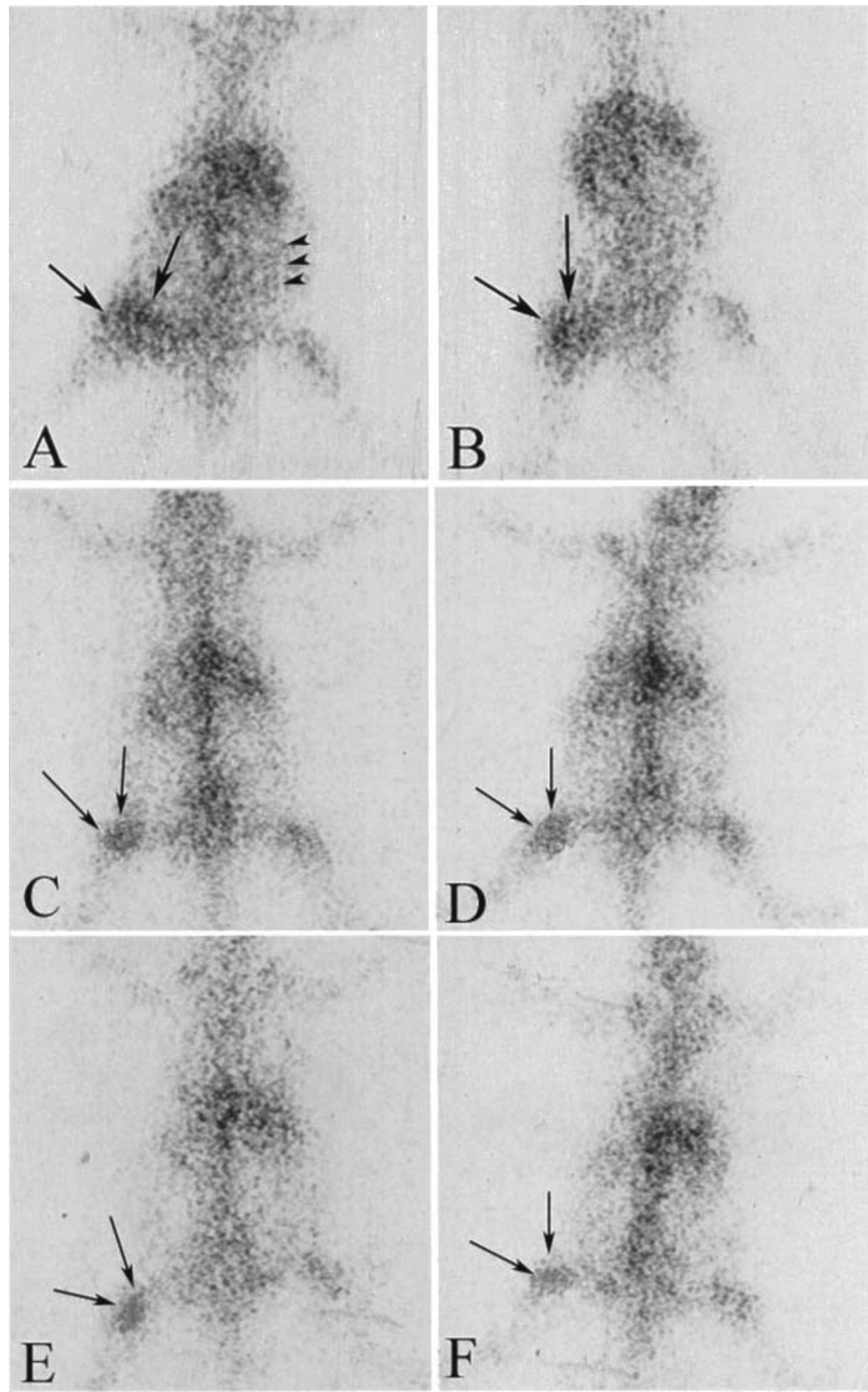
Table 2. The ratio of gallium uptake between infectious tissue and normal tissue after intramuscular injection of various organisms into the left caudal thigh of rats

\begin{tabular}{lcc}
\hline & \multicolumn{2}{c}{ L/N Ratio } \\
\cline { 2 - 3 } & $24 \mathrm{hr}$ & $48 \mathrm{hr}$ \\
\hline Control & $1.01 \pm 0.05$ & $1.04 \pm 0.04$ \\
S. aureus & $2.14 \pm 0.29$ & $2.00 \pm 0.40$ \\
E. coli & $1.27 \pm 0.16$ & $1.35 \pm 0.14$ \\
S. pneumoniae & $1.41 \pm 0.30$ & $1.48 \pm 0.34$ \\
\hline
\end{tabular}

Shown as MEAN \pm STD.

\section{RESULTS}

Hematologic results: The hematologic data after intramuscular injection of various micro-organisms into the left caudal thigh of rats are shown in Table 1. Intramuscular injection of these bacilli resulted in obvious change in the WBC population in the rats. The increase in WBC counts was most significant in the $S$. aureus group. The WBC counts increased from 7638 to 12682 counts $/ \mathrm{ml} 48 \mathrm{hr}$ after the injection. The increase in the WBC population was as high as $66 \%$. As for the other three groups, the increase was $49.1 \%$ for the $S$. pneumoniae group, $22.3 \%$ for the $E$. coli group and only $11 \%$ for the normal controls. The increase in PMNs was most significant in the rats with the $S$. aureus injection. The percentage of PMNs increased from 10.6\% to $39.7 \% 48 \mathrm{hr}$ after injection of $S$. aureus (an increase of $29.1 \%$ ). The rats with $S$. pneumoniae injection also showed an increase in the PMNs percentage (16\%) but no increase in PMNs was noted in either the E. coli or the control group.

Results of pathologic examination: Macroscopic analysis indicated infection of the injected sites in the three groups receiving bacilli, especially the $S$. aureus group. Abscess formation was noted, although no swelling or abscess formation was observed in the normal controls (Fig. 2). The microscopic pathological analysis showed that infiltration by inflammatory cells occurred in all three groups receiving the bacilli, but not in the control group. The infiltration by inflammatory cells was most significant in the $S$. aureus group and less significant in the E. coli group (Fig. 3).

Scintigraphic results: The $\mathrm{L} / \mathrm{N}$ ratios after intramuscular injection of various organisms into the left caudal thigh of the rats are shown in Table 2. The rats with $S$. aureus injection had significant gallium uptake at the infection site and the highest $\mathrm{L} / \mathrm{N}$ ratio of 2.14 on the 24 -hr image and 2.0 on the 48-hr image (Fig. 4). The rats with $S$. pneumoniae had the second highest $\mathrm{L} / \mathrm{N}$ ratio (1.41 at $24 \mathrm{hr}$ and 1.48 at 48 $\mathrm{hr}$ ). The $\mathrm{L} / \mathrm{N}$ ratio for the $E$. coli group was $1.27 \mathrm{at} 24 \mathrm{hr}$ and 1.35 at $48 \mathrm{hr}$. No obvious abnormal gallium uptake was demonstrated in the controls.

Statistical results: The statistical analysis of the hematological data and the ratio of gallium uptake in infected rats caused by various organisms is shown in Table 3. The 48hr WBC counts in the $S$. aureus group was significantly higher than those of the control, E. coli and S. pneumoniae groups. In addition, the $E$. coli and $S$. pneumoniae groups also had significantly higher 48-hr WBC counts than the control group. The 48-hr PMNs percentage in the $S$. aureus group was significantly higher than those of the control and $E$. coli groups but not statistically higher than the $S$. pneumoniae group. The 48-hr PMNs percentage in both E. coli and $S$. pneumoniae groups showed more statistical increase than that of the control group. The $\mathrm{L} / \mathrm{N}$ ratios for the $S$. aureus, E. coli and $S$. pneumoniae groups were significantly higher than that of the normal control on both 24-hr and 48$\mathrm{hr}$ images. In addition, the $S$. aureus group also had a significantly higher $\mathrm{L} / \mathrm{N}$ ratio than the $E$. coli and $S$. pneumoniae groups.

\section{DISCUSSION}

Ideally, an animal model should be inexpensive and easy to establish. SD rats are cheap, easy to obtain and easy to take care of, which makes it a good candidate for the creation of an infectious animal model. According to our findings, both $S$. aureus and $S$. pneumoniae were clearly highly invasive in SD rats. Both bacteria are gram positive but $E$. coli, a gram-negative pathogen, was less invasive in the SD rats. Among these three bacteria, S. aureus resulted in the most significant symptoms and signs of infection. The formation of an abscess on the site of injection, the increase in WBC and proportion of PMNs in the blood were significantly increased in this group compared to the controls. PMNs are mature phagocytes that migrate through tissues to destroy microbes and respond to inflammatory stimuli; their presence is always indicative of an acute infection. $S$. aureus infection is very common in hospitals. This pathogen can produce many toxins that cause illness in humans, sometimes leading to death $[11,12,19]$. S. aureus can adhere to the surface of endothelial cells and damage host cell membranes. This bacterium is of particular interest to microbiologists and public health officials because of outbreaks of antibiotic-resistant infections $[11,12]$. Therefore, $S$. aureus is an ideal bacterium for the creation of an animal

Fig. 4. Gallium scan (dorsal view) of the rats injected with various organisms. The first row is for the S. aureus group: (A) On the 24-hr image, significantly increased gallium uptake (arrows) at the infected left thigh with an $\mathrm{L} / \mathrm{N}$ ratio of 2.26 . In addition, physiologic bowel activity is also noted in the abdomen (arrow heads) (B) On the 48-hr image, the uptake of gallium in the infected thigh showed no apparent change with an L/N ratio of 2.35 (arrows). The second row is for the S. pneumoniae group: (C) On the 24-hr image, increased gallium uptake (arrows) in the infected left thigh with an L/N ratio of 1.69, (D) On the 48-hr image, the uptake of gallium in the infected thigh showed no apparent change with an $\mathrm{L} / \mathrm{N}$ ratio of 1.65 (arrows). The third row is for the E. coli: (E) On the 24-hr image, increased gallium uptake (arrows) in the infected left thigh with an L/N ratio of 1.42, (F) On the 48-hr image, the uptake of gallium in the infected thigh showed no apparent change with an $\mathrm{L} / \mathrm{N}$ ratio of 1.41 (arrows). 
Table 3. Statistical analysis of the hematological data and the ratio of gallium uptake in infected rats caused by various organisms

\begin{tabular}{lcccc}
\hline & Control & S. aureus & E. coli & S. pneumoniae \\
\hline WBC at $48 \mathrm{hr}$ & & & & \\
Control & - & 0.016 & 0.075 & 0.347 \\
S. aureus & 0.016 & - & 0.028 & 0.028 \\
E. coli & 0.075 & 0.028 & - & 0.916 \\
S. pneumoniae & 0.347 & 0.028 & 0.916 & - \\
\hline
\end{tabular}

\begin{tabular}{lcccc} 
PMNs at $48 \mathrm{hr}$ & & & & \\
Control & - & 0.047 & 0.753 & 0.251 \\
S. aureus & 0.047 & - & 0.015 & 0.174 \\
E. coli & 0.753 & 0.015 & - & 0.027 \\
S. pneumoniae & 0.251 & 0.174 & 0.027 & - \\
\hline
\end{tabular}

\begin{tabular}{lcccc} 
L/N ratio 24 hr & & & & \\
Control & - & 0.009 & 0.016 & 0.016 \\
S. aureus & 0.009 & - & 0.009 & 0.009 \\
E. coli & 0.016 & 0.009 & - & 0.464 \\
S. pneumoniae & 0.016 & 0.009 & 0.464 & - \\
\hline
\end{tabular}

\begin{tabular}{lcccc} 
L/N ratio $48 \mathrm{hr}$ & & & & \\
Control & - & 0.009 & 0.009 & 0.047 \\
S. aureus & 0.009 & - & 0.047 & 0.047 \\
E. coli & 0.009 & 0.047 & - & 0.464 \\
S. pneumoniae & 0.047 & 0.047 & 0.464 & - \\
\hline
\end{tabular}

model for investigating new infectious diagnostic methods or therapeutic drugs. The purpose of the study was to develop a small animal model of soft tissue infection without mortality which mimicked the clinical condition in humans.

Gallium imaging has been used for decades to detect infection clinically $[3,5,10,13,14,23]$. As gallium binds to transferrin, leukocyte lactoferrin, bacterial siderophores and inflammatory proteins, it can be used to localize foci of infection. In most infection areas, accumulation of gallium is always significant. In our study, the rats with the $S$. aureus injection had the highest gallium uptake at the infection site (thigh) when compared to those with S. pneumoniae and $E$. coli injection. These findings also corresponded to the results of blood tests and histopathologic examinations. Gallium citrate is not only accumulated in an infectious lesion but also in an inflammatory lesion $[11,12]$. In addition, Ga-67 citrate has been used as a tumor seeker for more than two decades and proven to be able to localize in a wide variety of tumors $[1,17]$. The uptake of gallium in the infection, inflammation or malignancy is not specific. Therefore, sometimes it is difficult to differentiate malignancy from infection or infection from inflammation only based on a gallium scan.

Moreover, physiological excretion of gallium into the intestines can also be a problem. The physiological bowel activity is sometimes difficult to differentiate from an intraabdominal infection and could cause a false positive gallium scan. There are two methods which may decrease the interference of normal gallium bowel activity during interpreta- tion: (1) cathartics and enemas, (2) serial imaging. Bowel preparation before gallium imaging has been reported to be useful in decreasing physical accumulation of gallium in the bowel $[3,8,18]$. Serial imaging is a useful method for distinguishing normal bowel activity from intra-abdominal infection. Normal gallium activity in the bowel will move with time, whereas an infectious lesion or abscess will appear unchanged.

In our study, all three pathogens, S. aureus, S. pneumoniae and $E$. coli, resulted in varying degrees of soft tissue infection in SD rats whereas the $S$. aureus had the best invasive ability in SD rats. We conclude that injection of $S$. aureus into the thigh of a SD rat is a good method to create soft tissue infection.

ACKNOWLEDGEMENT. This study was supported in part by a grant from the Taichung Veterans General Hospital Research Program (TCVGH-NCHU927609).

\section{REFERENCES}

1. Bar-Shalom, R., Mor, M., Yefremov, N. and Goldsmith, S. J. 2001. The value of Ga-67 scintigraphy and F-18 fluorodeoxyglucose positron emission tomography in staging and monitoring the response of lymphoma to treatment. Semin. Nucl. Med. 31: $177-190$.

2. Berns, J. S. 2003. Infection with antimicrobial-resistant microorganisms in dialysis patients. Semin. Dial. 16: 30-37.

3. Datz, F. L. 1996. Abdominal abscess detection: gallium, $111 \mathrm{In}-$ and $99 \mathrm{mTc}$-labeled leukocytes, and polyclonal and monoclonal antibodies. Semin. Nucl. Med. 26: 51-64.

4. Fischman, A. J., Rubin, R. H, Khaw, B. A., Callahan, R. J., Wilkinson, R., Keech, F., Nedelman, M., Dragotakes, S., Kramer, P. B. and LaMuraglia, G. M. 1988. Detection of acute inflammation with In-111 labeled nonspecific polyclonal IgG. Semin. Nucl. Med. 18: 335-344.

5. Forgacs, P., Wahner, H. W., Keys, T. F. and Van-Scoy, R. E. 1978. Gallium scanning for the detection of abdominal abscesses. Am. J. Med. 65: 949-954.

6. Gnanalingham, K. K., Elsaghier, A., Kibbler, C. and Shieff, C. 2003. The impact of methicillin-resistant Staphylococcus aureus in a neurosurgical unit: a growing problem. J. Neurosurg. 98: 8-13.

7. Gungor, F., Karayalcin, B., Gultekin, M., Paksoy, N., Senturk, U. K., Kaputlu, I., Yildiz, A. and Erkilic, M. 1996. Comparison of Tc-99m HIG and Ga-67 citrate in the evaluation of bacterial abscess in a rat model. Ann. Nucl. Med. 10: 79-83.

8. Hsieh, J. F., Que, J., Tsai, S. C., Cheng, K. Y., Lin, W. Y. and Wang, S. J. 2000. Does bowel preparation improve the quality of abdominal gallium scintigraphy? Nucl. Med. Commun. 21: 1033-1036.

9. Juweid, M., Fischman, A. J., Rubin, R. H., Baum, R. and Strauss, H. W. 1991. Comparison of Tc-99m labeled monoclonal antigranulocyte antibody and In-111 labeled IgG for the detection of focal sites of infection in rats. Nucl. Med. Commun. 12: 637-644.

10. Lin, W. Y., Chao, T. H. and Wang, S. J. 2002. Clinical features and gallium scan in the detection of post-surgical infection in the elderly. Eur. J. Nucl. Med. Mol. Imaging. 29: 371-375.

11. Lin, W. Y., Lan, J. L. and Wang, S. J. 1998. Gallium-67 Scintigraphy to Predict Response to Therapy in Active Lupus 
Nephritis. J. Nucl. Med. 39: 2137-2141.

12. Lin, W. Y., Lan, J. L., Cheng, K. Y. and Wang, S. J. 1998. Value of gallium-67 scintigraphy in monitoring the disease activity of lupus nephritis. Scand. J. Rheumatol. 27: 42-45.

13. Lin, W. Y., Wang, S. J., Cheng, K. Y., Shen, Y. Y. and Changlai, S. P. 1998. Diagnostic value of bone and Ga-67 imaging in skeletal tuberculosis. Clin. Nucl. Med. 23: 743-746.

14. McAfee, J. G. and Thakur, M. L. 1976. Survey of radioactive agents for in vitro labeling of phagocytic leukocytes. I. Soluble agents. J. Nucl. Med. 17: 480-487.

15. Moir, C. and Robins, R. E. 1982. Role of ultrasonography, gallium scanning, and computed tomography in the diagnosis of intra-abdominal abscess. Am. J. Surg. 143: 582-585.

16. Muroff, L. R. 1982. Detection of abdominal abscesses with gallium-67 citrate: correlative studies with computed tomography. Semin. Nucl. Med. 12: 173-183.

17. Nakahara, T., Togawa, T., Nagata, M., Kikuchi, K., Hatano, K., Yui, N. and Kubo, A. 2002. Detection and chemoradiotherapeutic evaluation of advanced esophageal squamous cell carcinoma using gallium-67 SPET: a preliminary study. Eur. $J$. Nucl. Med. Mol. Imaging. 29: 1072-1077.

18. Novetsky, G. J., Turner, D. A., Ali, A., Raynor, W. J. and Fordham, E. W. 1981. Cleansing the colon in gallium-67 scintigraphy: a prospective comparison of regimens. A .J. R. 137: 979-981.

19. Paterson, D. L., Rihs, J. D., Squier, C., Gayowski, T., Sagnimeni, A. and Singh, N. 2003. Lack of efficacy of mupirocin in the prevention of infections with Staphylococcus aureus in liver transplant recipients and candidates. Transplantation $\mathbf{7 5}$ : 194-198.

20. Peters, A. M., Danpure, H. J., Osman, S., Hawker, R. J., Henderson, B. L., Hodgson, H. J., Kelly, J. D., Neirinckx, R. D. and Lavender, J. P. 1986. Clinical experience with Tc-99m hexamethylprophyeneamineoxime for labelling leucocytes and imaging inflammation. Lancet 2: 946-949.

21. Rubin, R. H., Fischman, A. J., Needleman, M., Wilkinson, R., Callahan, R. J., Khaw, B. A., Hansen, W. P., Kramer, P. B. and Strauss, H. W. 1989. Radiolabeled, nonspecific, polyclonal human immunoglobulin in the detection of focal inflammation by scintigraphy: comparison with gallium- 67 citrate and technetium-99m-labeled albumin. J. Nucl. Med. 30: 385-389.

22. Sarda, L., Saleh-Mghir, A. S., Peker, C., Meulemans, A., Cremieux, A. C. and Le-Guludec, D. 2002. Evaluation of Tc-99m ciprofloxacin scintigraphy in a rabbit model of staphylococcus aureus prosthetic joint infection. J. Nucl. Med. 43: 239-245.

23. Tsai, S. C., Chao, T. H., Lin, W. Y. and Wang, S. J. 2002. Scintigraphic pattern of planar gallium scan in wound infection after colorectal surgery. Abdom. Imaging. 27: 442-447.

24. Welling, M. M., Lupetti, A., Balter, H. S., Lanzzeri, S., Souto, B., Rey, A. M., Savio, E. O., Paulusma-Annema, A., Pauwels, E. K. and Nibbering, P. H. 2001. Tc-99m labeled antimicrobial peptides for detection of bacterial and Candida albicans infection. J. Nucl. Med. 42: 788-794. 